\title{
Suivi de la pression interstitielle à l'œdomètre
}

A. RAHAL A. VUEZ

Laboratoire

de Géomécanique

Thermique et Matériaux

Institut National

des Sciences Appliquées

20, avenue des Buttes-de-

Coësmes

35043 Rennes Cedex

Les évolutions des pressions interstitielles au sein d'un massif de sol ou d'un échantillon œedométrique sont marquées par les conditions de chargement et de drainage. Pour traiter les problèmes de consolidation, et afin d'assurer la continuité entre condition initiale et conditions aux limites, il est nécessaire de prendre en compte des chargements fonction du temps. Deux types de chargement sont étudiés dans cet article : le chargement à vitesse constante «CRL » et le chargement cyclique sinusoïdal. Une solution analytique de l'équation de la consolidation établie en introduisant la vitesse de chargement est construite pour chacun de ces types de chargement. Puis une vérification expérimentale est faite, montrant la validité de la solution affichée. Les résultats remarquables sont d'une part, pour le premier type de chargement, la stabilisation de l'excès de pression interstitielle en fonction de la vitesse de chargement et. d'autre part pour le chargement sinusoïdal, la possibilité d'atteindre des excès de pression interstitielle de 1,14 fois la charge appliquée.

\section{Attended pore pressure in an œdemeter test}

The evolution of pore pressure inside a soil layer or an oedometer sample are influenced by loading and drainage conditions. To insure continuity within initial and boundarjes condition it is necessary to carry out consolidation with loading with respect to time. Two kinds of loading are presented in this paper : constant rate of loading a CRL $n$ and cyclic simusoidal loading. Each of them is first developed by means of an analytical solution of consolidation equation set up with the introduction of the rate of loading. Following the solution is validated by experiments. The main results are on one hand the stabilisation of excess pore pressure with respect to the rate of loading for constant rate of loading, and on the other hand the possibility to reach a level of pore pressure 1.14 time this one of the applied load for the sinusoidal loading. 


\section{Introduction}

Le chargement à la surface d'un massif de sol provoque en son sein une surpression du fluide interstitiel très préjudiciable pour la stabilité des ouvrages de construction. Cette surpression devient une préoccupation du concepteur. Il doit la prendre en compte dans différents types de problème teis que l'évaluation des temps de tassement ou bien les vérifications de la stabilité d'ouvrages qu'ils soient de fondation, de soutènement ou encore de construction en terre. En effet, le calcul de stabilité ne peut être mené de manière satisfaisante que si l'on peut évaluer la valeur de la surpression interstitielle. Dans l'analyse de ces situations de calcul, le projeteur fait appel à la théorie de la consolidation comme une aide à la prévision. Or l'usage classique de cette théorie s'appuie sur une solution construite à partir des hypothèses simplificatrices relatives à une couche de sol fin horizontale suivantes: 1) condition initiale : l'excès de pression interstitielle est égal à l'accroissement de contrainte dû au chargement du massif de sol

2) conditions aux limites de drainage double face, c'està-dire pression interstitielle nulle sur les faces de la couche de terrain ou de l'échantillon dans le cas de l'essai œdométrique.

En pratique, l'application d'un chargement n'est jamais instantanée. Pour mieux correspondre à la réalité physique, il est préférable de choisir une solution mathématique assurant une continuité de la valeur de la pression entre condition initiale et conditions aux limites. Pour ne pas obtenir une solution triviale, il est alors nécessaire d'introduire la vitesse d'application du chargement dans l'équation de la consolidation. Ce type de solution a déjà été introduit par Aboshi et al. (1971) lorsqu'ils étudièrent les chargements continus de type (C Constant Rate of Loading », appelé encore chargement à vitesse de chargement constante (c CRL 》.

Nous étudions dans ce qui suit deux types de chargement continu : le chargement monotone croissant uniformément et les cycles de charge-décharge dont la période est suffisamment grande pour négliger les effets d'inertie. Ce cas très particulier de chargement cyclique induit des atténuations et des déphasages entre chargement et pression interstitielle qui génèrent des excès de pression interstitielle instantanés très supérieurs au chargement et donc très préjudiciables à la stabilité des ouvrages.

La condition de chargement fonction du temps en surface peut prendre des formes quelconques, pas uniquement linéaires. Des exemples se rencontrent dans le cas de variation saisonnière de niveau de nappe phréatique ou bien dans celui de stockage de matières pondérales sur de grandes surfaces. On obtient alors des cycles de charge décharge dont la période atteint plusieurs mois. Même à cette cadence, le phénomène cyclique engendre des fluctuations importantes de la pression interstitielle.

\section{Mise en équation du phénomène de consolidation}

La mise en équation du processus de consolidation est comparable à celle de tout phénomène de diffusion. L'équation de continuité sur le fluide est écrite en introduisant la loi de Darcy comme loi d'écoulement des particules de fluide par rapport aux grains. En condition unidirectionnelle suivant $\mathrm{z}$, elle s'énonce ainsi (Berry et Poskitt, 1972 ; Vuez et Rahal, 1994)

$$
\frac{\partial \varepsilon_{v}}{\partial t}+\frac{n}{\rho_{w}} \frac{\partial \rho_{w}}{\partial t}=\frac{1}{\rho_{w} g} \frac{\partial}{\partial z}\left(k \frac{\partial u}{\partial z}\right)
$$

où $\varepsilon$, représente la déformation volumique de la matrice du sol;

n la porosité ;

$P_{w}$ la masse volumique de l'eau;

g l'accélération de la pesanteur ;

k la perméabilité du milieu :

$u$ la pression du fluide interstitiel.

La résolution de cette équation est faite en prenant la pression u comme variable principale. Il est alors nécessaire d'écrire les équations d'état du squelette du sol, celle du fluide interstitiel et l'équation liant contrainte effective $\sigma$ à la contrainte appliquée $\sigma$, soit : $\varepsilon_{v}=m_{v} \Delta \sigma \quad$ avec $m_{v}$ coefficient de compressibilité du squelette ;

$d \rho_{w}=-\rho_{w} \beta d u$ avec $\beta$ coefficient de compressibilité du fluide interstitiel

$\sigma^{\prime}=\sigma-\mathrm{u} \quad$ d'après le postulat de Terzaghi.

L'équation (1) prend alors la forme suivante, Rahal (1993) :

$$
\frac{1}{c_{v}} \frac{\partial u}{\partial t}=\frac{\partial^{2} u}{\partial z^{2}}+\frac{1}{c_{v g}} \frac{\partial \sigma}{\partial t}
$$

relation dans laquelle les coefficients de consolidation $\mathrm{c}_{\mathrm{v}}$ et $\mathrm{c}_{\mathrm{vg}}$ sont explicités par :

$$
c_{v}=\frac{k}{\rho_{w} g\left(n \beta+m_{v}\right)} \text { et } c_{v g}=\frac{k}{\rho_{w} g m_{v}}
$$

La solution retenue de l'équation (2) est celle correspondant aux conditions expérimentales d'un milieu de hauteur $\mathrm{H}$, drainé simple face (Ozisik, 1980), conditions que l'on retrouve dans l'essai de compressibilité à l'œdomètre avec mesure de la pression interstitielle : 1) condition initiale, la pression $u$ est constante en fonction de $\mathrm{z}: \mathrm{u}(\mathrm{z}, 0)=\mathrm{u}_{0}$; 2) conditions aux limites telles que : $\frac{\partial u}{\partial z}(0, t)=0$ et
$u(H, t)=f(t)$.

Si on appelle $g(z, t)$ la fonction $\frac{\partial \sigma}{\partial t}$, alors la solution s'ecrit :

$$
\begin{aligned}
& u(z, t)=\frac{2}{H} \sum_{m=1}^{\infty} \cos \frac{M z}{H} e^{-M^{2} T_{v}} \int_{0}^{H} \cos \frac{M z}{H} u_{0} d z \\
& +\frac{c_{v}}{c_{v g}} \frac{2}{H} \sum_{m=1}^{\infty} \cos \frac{M z}{H} e^{-M^{2} T_{v}} \int_{0}^{t} e^{-M^{2} T_{v}} \int_{0}^{H} \cos \frac{M z}{H} g\left(z, t^{\prime}\right) d z d t^{\prime}
\end{aligned}
$$


$+c_{v} \frac{2}{H^{2}} \sum_{m=1}^{\infty} \cos \frac{M z}{H} e^{-M^{2} T_{v}} M \sin M \int_{0}^{t} e^{-M^{2} T v} f\left(t^{\prime}\right) d t^{\prime}$

avec: $\quad M=(2 m-1) \frac{\pi}{2}$ et $T_{v}=\frac{c_{v} t}{H^{2}}$

C'est cette solution (4) qui est utilisée dans ce quí suit pour étudier l'évolution de la pression interstitielle dans le cas de chargements linéaire et sinusoïdaux obtenus par variation de la contrainte appliquée en surface de couche compressible ou en tête d'un échantillon œedométrique.

\section{3}

\section{Solutions de l'équation de la consolidation dans le cas d'un chargement fonction du temps}

Le chargement linéaire a déjà été étudié par de nombreux auteurs dans le but de rendre compte de la durée de construction sur la croissance de la pression interstitielle dans la couche de fondation. Pourtant son utilisation est surtout intéressante dans le cas de l'essai œdométrique car il permet une détermination plus rapide du coefficient de consolidation $\mathrm{c}_{\mathrm{v}}$.

Le chargement sinusoïdal est moins décrit dans la littérature. Néanmoins il conduit à des observations remarquables, si on les confronte à la réalité des constructions.

\section{3,1}

\section{Chargement continu suivant une rampe}

La condition de chargement en contrainte totale s'écrit :

$$
\sigma=R t+\sigma_{0}
$$

relation dans laquelle : $\mathrm{R}$ exprime le taux de chargement;

$\sigma_{0}$ le chargement à l'instant initial.

On en déduit la fonction $\mathrm{g}(\mathrm{z}, \mathrm{t})$ :

$$
g(z, t)=\frac{\partial \sigma}{\partial t}=R
$$

Les conditions initiale et aux limites sont les suivantes :

$$
\begin{array}{ll}
\text { pour } \mathrm{t}=0 & \mathrm{u}_{0}(\mathrm{z}, 0)=\mathrm{u}_{0} \\
\text { pour } \mathrm{z}=\mathrm{H} & \mathrm{u}(\mathrm{H}, \mathrm{t})=\mathrm{f}(\mathrm{t})=\mathrm{u}_{0}
\end{array}
$$

La solution (4) s'écrit alors :

$$
u(z, t)=u_{0}+2\left(1-n_{c t}\right) \frac{R \cdot t}{T v} \sum_{m=1}^{\infty} \cos \frac{M z}{H} \frac{\sin M}{M^{3}}\left(1-e^{-M^{2} T_{v}}\right)
$$

Le coefficient $n_{c}$ représente la quantité :

$$
n_{c f}=\frac{n \beta}{m_{v}+n \beta}=1-\frac{c_{v}}{c_{v g}}
$$

Il traduit l'état comparatif, en terme de compressibilité, entre les deux constituants du sol : squelette du sol et fluide interstitiel.

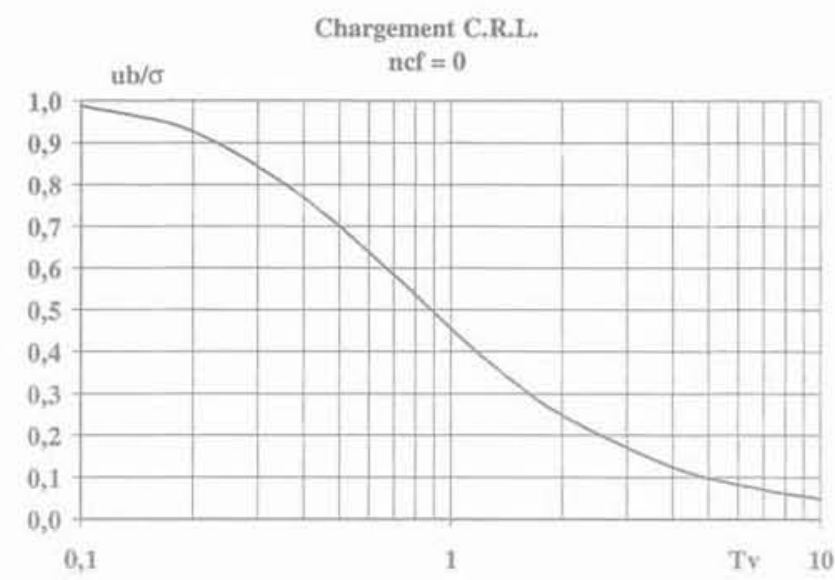

FIG.1 Rapport de la pression interstitielle sur la charge $-\mathrm{n}_{\mathrm{cf}}=0$.

Ratio of the pore pressure upon the load $-n_{\mathrm{g}}$ $=0$.

Le tracé du rapport $\frac{\mathrm{u}(0, \mathrm{t})}{\sigma(\mathrm{t})}$ en fonction du rapport

Tv est donné sur la figure 1 pour $n_{c}$ égal à zéro. Les tracés pour d'autres valeurs de $n_{\mathrm{d}}$ différentes de zéro se déduisent de la première courbe par affinité de rapport $\left(1-n_{c}\right)$.

\section{2}

\section{Chargement sinusoïdal}

Les conditions initiales et aux limites pour ce type de chargement sont les suivantes :

$\sigma=A p \sin \omega t+\sigma_{0}$ d'où $g(z, t)=\frac{\partial \sigma}{\partial t}=A p \omega \cos \omega t$ $\mathrm{u}_{0}(\mathrm{z}, 0)=\mathrm{u}_{0} \quad ; \quad \mathrm{u}(\mathrm{H}, \mathrm{t})=\mathrm{f}(\mathrm{t})=\mathrm{u}_{0}$

La solution (4) s'écrit dans ce cas :

$$
\begin{gathered}
u(z, t)=u_{0}+2 A p\left(n_{c t}-1\right) \sum_{m=1}^{m=} \frac{(-1)^{m}}{M+\theta_{v}^{2} M^{5}} \\
\cos \frac{M z}{H}\left(\sin \omega t+M^{2} \theta_{v} \cos \omega t-M^{2} \theta_{v} e^{-M^{2} T_{v}}\right)
\end{gathered}
$$

avec: $\quad \theta_{v}=\frac{c_{v}}{\omega H^{2}}$ et $n_{c f}=\frac{n \beta}{m_{v}+n \beta}=1-\frac{c_{v}}{c_{v g}}$

Le coefficient $\theta_{\text {v }}$ est analogue au facteur temps $\mathrm{T}_{v}$. I est fonction de la qualité du terrain, de celle du fluide interstitiel, de la géométrie du milieu et de la fréquence du chargement.

Après établissement du régime stationnaire, l'expression de la pression interstitielle pour $\mathrm{z}=0$, notée par la suite $u_{b}$, peut alors se mettre sous la forme:

$$
u_{b}=u_{0}+A p_{u b} \sin \left(\omega t+\phi_{u b}\right)
$$

L'amplitude $A p_{u b}$ de la pression $u_{b}$ est atténuée par rapport à celle du chargement Ap. Le coefficient d'atténuation est défini par :

$$
A t t_{u b}=\frac{A p u b}{A p}
$$

De même le déphasage de la pression $u_{b}$ par rapport au chargement est exprimé par le terme Déph mesuré en unité temps ou bien par son correspondant 
adimensionnel, si T représente la période de chargement :

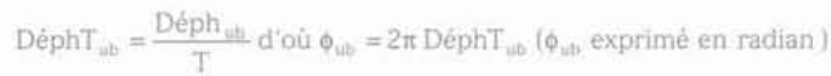

Il est intéressant de tracer les variations de l'atténuation Att en fonction du coefficient $\theta_{v}$ pour des différents taux de compressibilité du fluide $n_{\text {. }}$ La figure 2 donne la courbe représentative pour $n_{f}=0$. Les variations pour d'autres valeurs de $n_{\text {, }}$ se déduisent de cette courbe par affinité de rapport $\left(1-n_{f}\right)$. La valeur zéro pour $n$ représente le cas particulier d'un fluide incompressible. Cette hypothèse est souvent avancée dans les calculs d'ouvrage en mécanique des sols.

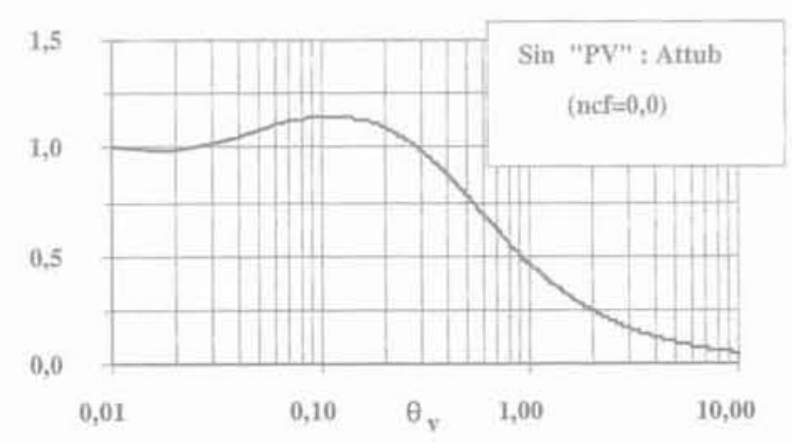

FG.2 Atténuation de la pression interstitielle $u_{b}$. Pore pressure $u_{b}$ attenuation.

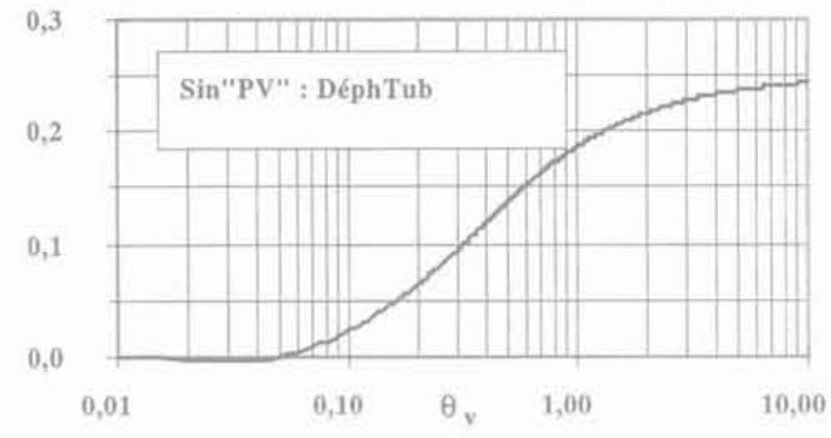

FG, 3 Déphasage de la pression interstitielle $u_{b}$. Out of phase of pore pressure $u_{b}$.

La figure 3 représente la variation du déphasage Déph $T_{u b}$ en fonction de $\theta_{v}$. Il ne dépend pas de $n_{c}$.

A l'examen de ces courbes, deux observations peuvent être faites :

- d'abord l'amplitude de la pression interstitielle peut dépasser celle du chargement pour des valeurs de $\theta_{\mathrm{v}}$ comprises entre 0,03 et 0,3 ;

- ensuite la pression interstitielle $\mathrm{u}_{\mathrm{b}}$ admet un déphasage en avance par rapport à la marche du chargement à partir d'un $\theta_{\mathrm{y}}$ de 0,05 .

La première observation est analysée par la suite au moyen de l'évolution des isochrones des surpressions interstitielles. Elle est confirmée par des résultats expérimentaux obtenus lors d'un chargement à l'œdomètre dont nous décrivons les caractéristiques dans le paragraphe suivant.

\section{Matériel expérimental}

Les essais ont été réalisès au moyen d'une cellule œdométrique avec mesure de pression interstitielle. L'échantillon est donc enfermé dans une bague rigide, imperméable, avec la face supérieure ouverte sur un dispositif de pression (noté par la suite contre-pression). La face inférieure est appuyée sur une embase dans laquelle prend place un capteur de pression surmonté d'une pierre poreuse. Un vérin dont on contrôle la pression tout en mesurant le déplacement, appuie sur la face supérieure de l'éprouvette. Une pierre poreuse est intercalée entre l'éprouvette et la plaque d'appui du vérin. La caractéristique essentielle de l'appareillage est de permettre la mesure de la pression du fluide interstitiel en même temps que celle de la déformation de l'échantillon.

La figure 4 donne un schéma de principe de l'essai cedométrique et du montage de l'échantillon dans la cellule.

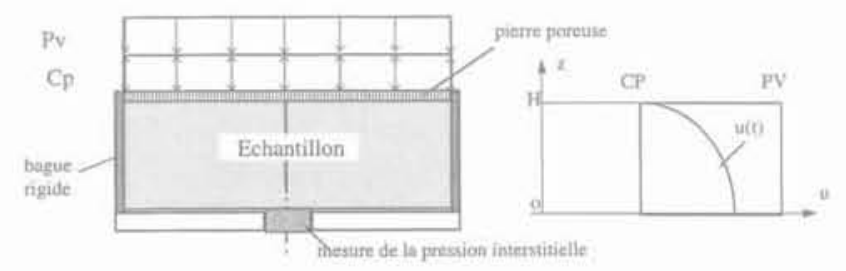

ตุ.4 Schéma du montage de l'essai. Edometer test.

Les dimensions de l'éprouvette choisies dans le cadre de nos essais sont:

- un diamètre $\mathrm{D}$ de $50 \mathrm{~mm}$;

- une hauteur $\mathrm{H}$ de $10 \mathrm{~mm}$ à $12 \mathrm{~mm}$.

La sollicitation sur l'échantillon de sol est exercée soit par un vérin appuyant sur le dessus de l'échantillon, soit par une mise en pression du fluide interstitiel de l'échantillon : chargement par contre-pression. Le contróle du chargement est effectué par la mesure de la pression dans le vérin et par celle de la contre-pression. La réponse de l'échantillon à la sollicitation est suivie par la mesure de la pression au bas de l'échantillon, et de la variation de hauteur de l'échantillon. La recherche de la quasi simultanéité des mesures de ces quatre grandeurs, pour mieux apprécier les retards entre déformation et dissipation de la pression interstitielle, nécessite l'usage de capteurs électriques et d'une chaine d'acquisition. La réalisation de chargements en fonction du temps tels que chargements continus ou chargements sinusoïdaux impose un contrôle de l'essai par un automate. L'acquisition des données et le pilotage des essais ont été réalisés à l'aide d'un système d'acquisition (appareillage « Keithley 570 ») piloté par un micro-ordinateur déroulant un programme modulaire de gestion de l'essai.

Le décalage de la déformation par rapport à la dissipation de la pression est ainsi perceptible mais il ne pourra être analysé que si on tient compte des difficultés de mesures. Plusieurs effets ont été étudiés par Rahal (1993), qui sont :

- les déplacements parasites ayant pour origine la 
déformation propre de la cellule ; après étalonnage, ils ont été défalqués des mesures brutes (erreurs systématiques) :

- les délais de mesure de la pression à cause de la « respiration ») du capteur. Le calcul montre que le temps nécessaire à la mesure de la pression sur le capteur est très inférieur $\left(<10^{-3} \mathrm{~s}\right)$ au temps de drainage ;

- la synchronisation des chargements entre vérin et pression. Un chargement de $20 \mathrm{kPa}$ est obtenu au bout de 0,3 s sur le vérin et de 1 s pour la contre-pression. Ceci constitue une limitation du dispositif expérimental pour le choix de la vitesse de chargement.

\section{5}

\section{Résultats expérimentaux de chargement suivant une rampe}

Le chargement «CRL » a été observé sur un sol en provenance de Claye-Souilly. Les caractéristiques du matériau sont résumées dans le tableau I ci-dessous.

TABLEAU Caractéristiques du sol de Claye-Souilly. Characteristics of Claye-Souilly soil.

\begin{tabular}{l|c|c} 
& Echantillon 3 & Echantilion 5 \\
\hline $\begin{array}{l}\text { Poids volumique humide } \\
\left(\mathrm{kN} / \mathrm{m}^{3}\right)\end{array}$ & 20,85 & 21,59 \\
\hline $\begin{array}{l}\text { Teneur en eau à la mise } \\
\text { en place }(\%)\end{array}$ & 11,2 & 15,0 \\
\hline $\begin{array}{l}\text { Poids volumique des grains } \\
\left(\mathrm{kN} / \mathrm{m}^{3}\right)\end{array}$ & 26,4 & 26,4 \\
\hline indice des vides initial & 0,410 & 0,408 \\
\hline Valeur au bleu & 2,4 & - \\
\hline
\end{tabular}

L'échantillon $n^{\circ} 5$ testé avait pour hauteur $11 \mathrm{~mm}$. La contre-pression était de $342 \mathrm{kPa}$. Le chargement effectif en fin de rampe a été fixé au voisinage de $15 \mathrm{kPa}$. Les conditions d'essais sont résumées dans le tableau II.

Quatre tests ont été effectués pour des vitesses de chargement variant de 0,00108 à $0,00707 \mathrm{kPa} / \mathrm{s}$. La pres-

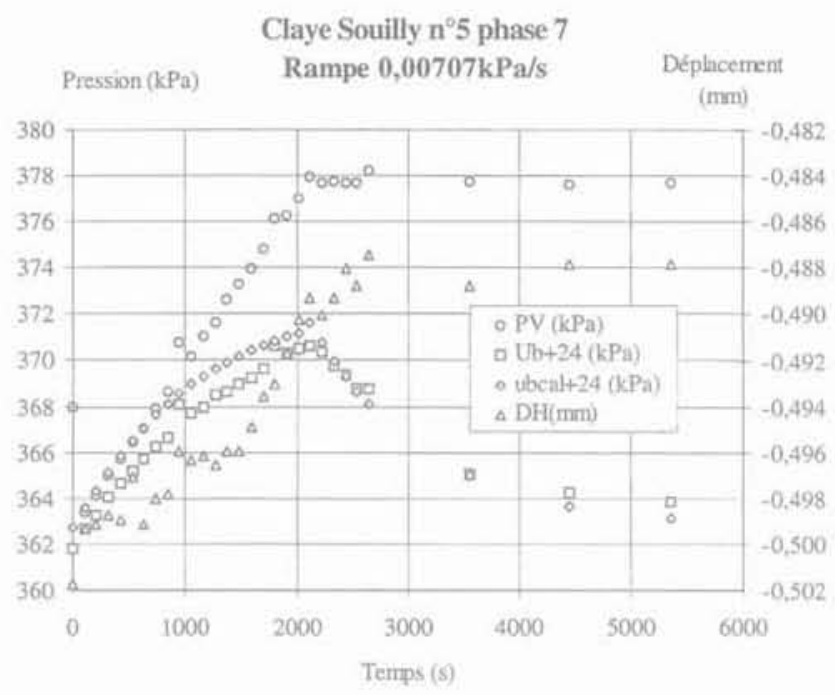

FiG. 5 Essai à chargement «CRL » matériau de Claye-Souilly $n^{\prime \prime} 5$.

"CRL $n$ test. Claye-Souilly clay $n^{\circ} 5$

sion sur le vérin est notée par la suite «PV ». La figure 5 représente, pour une valeur de la vitesse de chargement de $0,00707 \mathrm{kPa} / \mathrm{s}$, d'une part, les variations de la pression sur le vérin ou grandeur régulée «PV ») et, d'autre part, les valeurs des grandeurs mesurées : pression interstitielle au bas de l'échantillon « ub » et déplacement en tête «DH . Les quatre vitesses de chargement ont été choisies pour obtenir des valeurs distinctes et significatives des rapports de la pression interstitielle à la charge. Le chargement maximal appliqué a été limité à $15 \mathrm{kPa}$ pour réduire les effets de la variation de la compressibilité du sol en fonction de la contrainte effective et pour rester dans les mêmes ordres de grandeur que les amplitudes utilisées dans les essais à chargements sinusoïdaux.

La stabilisation de l'excès de pression interstitielle observée est semblable à celle décrite par le calcul. Les écarts sont inférieurs à $1 \mathrm{kPa}$.

Pour chacun des chargements, le coefficient de consolidation est calculé en déterminant le facteur temps $T_{y}$ en portant la valeur du rapport de la pression interstitielle à la charge sur l'abaque de la figure 1. Les résultats sont reportés dans le tableau II. On constate, comme l'avait déjà décrit Peignaud (1975), que le coefficient de consolidation augmente avec la vitesse de chargement.

TARLAU II Conditions d'essai de chargement "CRL » et résultats. Test conditions and results for «CRL $»$ loading.

\begin{tabular}{|c|c|c|c|c|c|}
\hline $\begin{array}{l}\text { Numéro du } \\
\text { chargement }\end{array}$ & $\begin{array}{c}\text { Chargement } \\
\text { total effectif } \\
\text { ( (KPa) }\end{array}$ & $\begin{array}{l}\text { Rampe } \mathrm{B} \\
(\mathrm{kPa} / \mathrm{s})\end{array}$ & $\begin{array}{l}\text { Termps de } \\
\text { chargement to } \\
\text { (s) }\end{array}$ & $\begin{array}{l}\text { Pression } \\
\text { interstitielle } \\
\text { maximale } \\
\text { (kPa) }\end{array}$ & $\begin{array}{c}\text { Coefficient de } \\
\text { consolidation } \\
c_{c} \\
\left(\mathrm{~m}^{2} / \mathrm{s}\right)\end{array}$ \\
\hline 511-phase 9 & 13,4 & 0,00108 & 13900 & 2,6 & $2,510^{-8}$ \\
\hline 511-phase 4 & 14,6 & 0,00225 & 6327 & 4,4 & $3.510^{-8}$ \\
\hline 511 phase 8 & 13,0 & 0,00375 & 4000 & 5.6 & $3,8 \quad 10^{-8}$ \\
\hline 511 phase 7 & 14,5 & 0,00707 & 2120 & 8,4 & $4,7 \quad 10^{-8}$ \\
\hline
\end{tabular}


Les écarts sur la valeur du coefficient de consolidation peuvent être interprétés par la non-linéarité du comportement du sol en compression. L'étude du comportement non linéaire a été abordée par Vaid (1985). Ce dernier a exprimé une solution analytique de l'évolution de la pression interstitielle en exprimant la compressibilité du squelette en fonction de la contrainte $\sigma$ par une loi hyperbolique. Cette solution est paramétrée par un coefficient appelé B et défini par:

$$
\mathrm{B}=\frac{R H^{2}}{\sigma_{0} c_{y}},
$$

Elle s'écrit :

$$
\frac{\mathrm{u}(\mathrm{z}, \mathrm{t})}{\mathrm{Rt}}=\frac{\left(1+B T_{v}\right)}{B T_{i}}\left\{1-\exp \left(-\frac{4}{\pi} \sum_{m=1}^{\tilde{\Sigma}} \cos \frac{M z}{H} \sin M \int_{0}^{T} \frac{e^{-n^{2} p^{2} \pi} \pi_{y}-t / 4}{1+B \tau} B d \tau\right)\right\}
$$

Le calcul montre que les écarts par rapport au comportement linéaire sont significatifs pour des valeurs de $\mathrm{B}$ supérieures à l'unité. Or la valeur de B calculée dans les essais décrits ci-dessus est inférieur à 0,7 . On en déduit alors que la non-linéarité n'explique pas les écarts observés. Les causes peuvent être recherchées dans le comportement viscoélastique du matériau.

Tant l'expérimentation que la modélisation par calcul montrent une stabilisation de l'excès de pression interstitielle en fonction de la vitesse de chargement. Cette remarque est applicable à un massif de sol soumis à un chargement de surface tel que la mise en place d'un remblai ou d'une construction. En effet la réalisation d'un remblai ne s'effectue pas instantanément mais demande un délai de construction pendant lequel les travaux sont effectués à une cadence que l'on peut assimiler à une vitesse de construction. La cadence choisie conduit à un majorant de l'excès de pression interstitielle que l'on peut situer par rapport à la condition de rupture par cisaillement soit du sol de fondation, soit du corps du remblai d'une digue par exemple.

\section{6}

\section{Dépouillement des chargements sinusoïdaux}

Les essais de chargement sinusoïdaux ont été effectués sur le même sol, en provenance de Claye-Souilly, dont les caractéristiques ont été données dans le tableau I du paragraphe 5 .

Les caractéristiques des échantillons et des chargements sont résumés dans le tableau III. Les résultats expérimentaux sur la réponse de la pression interstitielle sont représentés respectivement sur les figures 7 et 8 pour des périodes de 60 s et 1800 s.

Les valeurs du coefficient de consolidation $c_{v}$ sont obtenues à partir de la mesure du déphasage de la pression interstitielle. Il faut noter que celui-ci ne dépend pas du coefficient $n_{\mathrm{c}}$ (cf. Fig. 3). Connaissant le coefficient de consolidation $\mathrm{c}_{\mathrm{y}}$ et au moyen de la mesure de l'atténuation, il est possible de calculer le coefficient $\mathrm{n}_{\text {cr }}$

L'examen des résultats présentés par le tableau III montre deux faits intéressants : d'abord le dépassement de la charge par la pression interstitielle et ensuite la variation du coefficient $\mathrm{n}_{\mathrm{c}}$ avec la période.

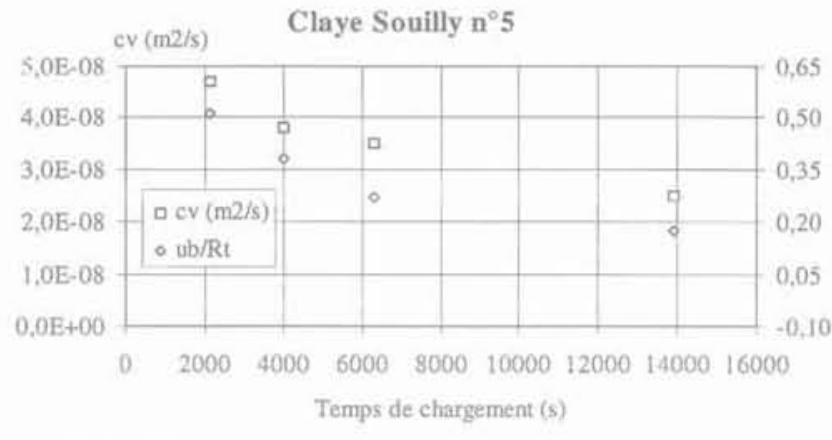

FG. 6 Évolution du $\mathrm{C}_{\mathrm{v}}$ et du maximum de ub à la fin de la rampe d'un chargement «CRL »Claye-Souilly $n^{\circ} 5$.

Evolution of $\mathrm{C}$ and the maximum of $\mathrm{ub}$ at the end of the rampe of "CRL $x$ test.

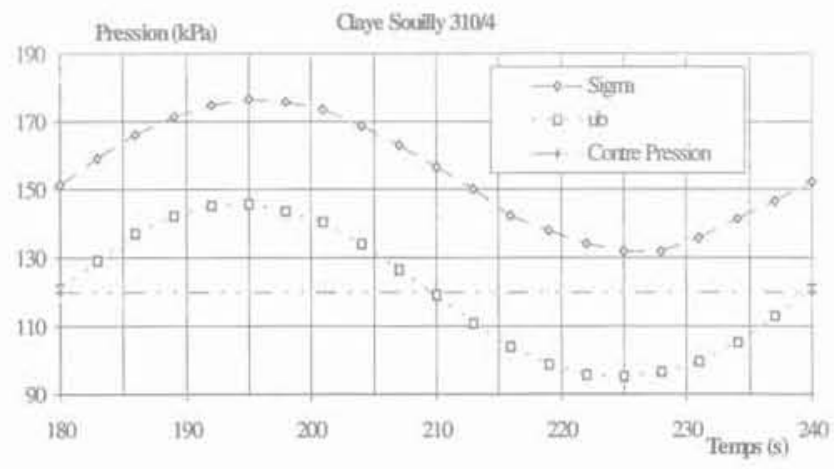

Fic. 7 Évolution de la charge $\sigma$ et de la pression interstitielle $u_{b}$.

Échantillon de Claye-Souilly/phase 310/4 Loading and excess pore pressure. ClayeSouilly sample/stage $310 / 4$.

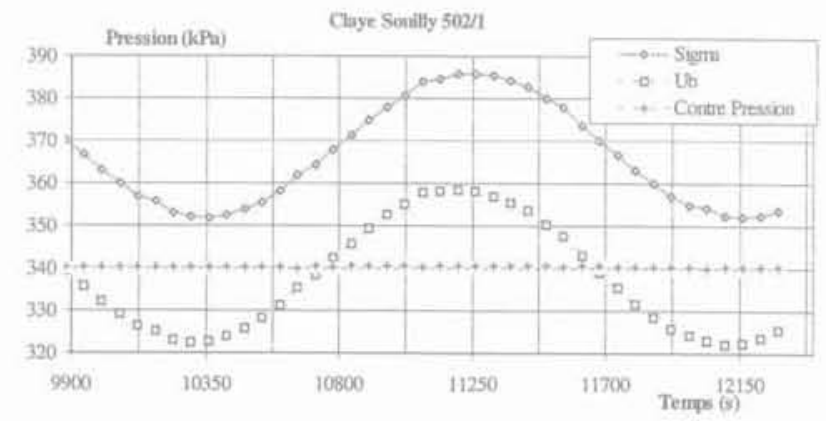

F16.8 Évolution de la charge $\sigma$ et de la pression interstitielle $u_{b}$.

Échantillon de Claye-Souilly/phase 502/1. Loading and excess pore pressure - ClayeSouilly sample/stage $502 / 1$.

\section{1}

\section{Atténuation supérieure à l'unité}

L'atténuation Attu est supérieure à l'unité dans six conditions de chargement, avec des dépassements pouvant atteindre $13 \%$. Ceci traduit une surpression interstitielle supérieure au chargement en contrainte 
TABLEAU III Caractéristiques des échantillons et conditions de chargement. Dépouillement des chargements sinusoïdaux.

Sample characteristics and loading conditions. Analysis of sinusoïdal loadings.

\begin{tabular}{|c|c|c|c|c|c|c|c|c|c|}
\hline Échantillon & $N^{\circ}$ phase & $\begin{array}{l}\text { Hauteur } \\
\text { (m) }\end{array}$ & $\begin{array}{l}\text { Indice } \\
\text { de } \\
\text { vide }\end{array}$ & $\begin{array}{l}\text { Périodé } \\
\text { (s) }\end{array}$ & Attu & Déph & $\theta_{v}$ & $\left(\mathrm{~m}^{2} / \mathrm{s}\right)$ & $n_{c}$ \\
\hline Claye 3 & $310 / 4$ & 0,0112 & 0,340 & 60 & 1,126 & 0,03 & 0,115 & $1,5 e-06$ & 0,018 \\
\hline Claye 5 & $501 / 5$ & 0,0103 & 0.319 & 60 & 0,941 & $-0,075$ & 0,050 & $5,6 e-07$ & 0,227 \\
\hline Claye 5 & $501 / 21$ & 0,0100 & 0,282 & 300 & 1,042 & 0 & 0,050 & $1,0 \mathrm{e}-07$ & 0,039 \\
\hline Claye 5 & $501 / 23$ & 0,0100 & 0,283 & 900 & 1,052 & 0 & 0,050 & $3,5 e-08$ & 0,030 \\
\hline Claye 5 & $502 / 2$ & 0,0101 & 0,290 & 1800 & 1,079 & 0,023 & 0,100 & $3,5 e-08$ & 0,059 \\
\hline Claye 5 & $502 / 3$ & 0,0101 & 0,289 & 7200 & 0,874 & 0,077 & 0,238 & $2,1 e-08$ & 0,170 \\
\hline Claye 5 & $503 / 2$ & 0,0101 & 0,291 & 1200 & 1,091 & 0,012 & 0,075 & 4,0e-08 & 0,036 \\
\hline Claye 5 & $504 / 2$ & 0,0101 & 0,293 & 3600 & 1,003 & 0,079 & 0,215 & $3,8 \mathrm{e}-08$ & 0,071 \\
\hline Claye 5 & $504 / 4$ & 0,0101 & 0.293 & 5400 & 0,915 & 0,086 & 0,266 & $3,2 \mathrm{e}-088$ & 0,106 \\
\hline Claye 5 & $505 / 2$ & 0,0101 & 0,293 & 10800 & 0,764 & 0,105 & 0,339 & $2,0 \mathrm{e}-08$ & 0,189 \\
\hline Claye 5 & $506 / 2$ & 0,0101 & 0,294 & 9000 & 0,803 & 0,100 & 0,315 & $2,2 \mathrm{e}-08$ & 0,172 \\
\hline Claye 5 & $506 / 3$ & 0,0101 & 0,294 & 18000 & 0,573 & 0,129 & 0,450 & $1,6 \mathrm{e}-08$ & 0,305 \\
\hline Claye 5 & $507 / 3$ & 0,0101 & 0,296 & 7200 & 0,889 & 0,083 & 0,257 & $2,3 e-08$ & 0,141 \\
\hline Claye 5 & $508 / 2$ & 0,0101 & 0,291 & 36000 & 0,385 & 0,157 & 0,635 & $1,1 e-08$ & 0,419 \\
\hline Claye 5 & $508 / 3$ & 0,0101 & 0,293 & 72000 & 0,218 & 0,192 & 1,100 & $1,0 \mathrm{e}-08$ & 0,489 \\
\hline Claye 5 & $509 / 2$ & 0,0101 & 0,294 & 54000 & 0,284 & 0,172 & 0,785 & $0,9 e-08$ & 0,489 \\
\hline
\end{tabular}

totale. Cette situation est prévue par la théorie (cf. Fig. 2).

On l'observe avec l'échantillon Claye 3 pour une période de 60s (cf. Fig. 7), et avec l'échantillon Claye 5 pour une période de 1800 s (cf. Fig. 8). En effet, les périodes ne sont pas identiques car les deux échantillons ont un coefficient de consolidation différent.

Les résultats expérimentaux de la phase $310 / 4$ sont confrontés aux résultats calculés par l'expression (6) avec un coefficient $\theta_{\mathrm{v}}$ de 0,11 et un taux $\mathrm{n}_{\mathrm{g}}$ de 0,018 . La figure 9 montre la bonne concordance.

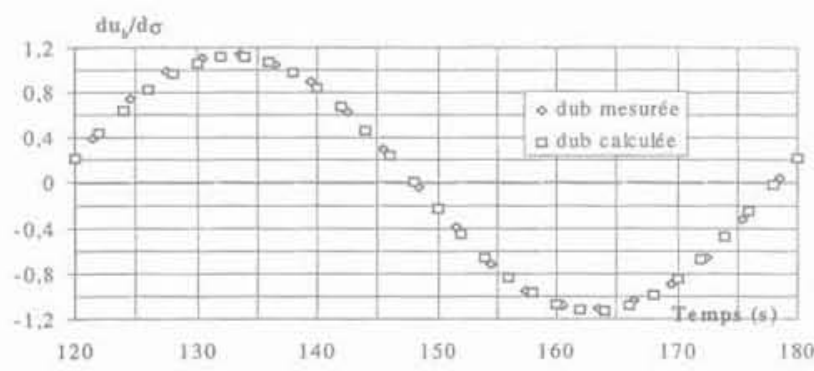

5G. 9 Comparaison entre la pression interstitielle $u_{b}$ mesurée et celle calculée. Échantillon de Claye-Souilly/phase 310/4 Comparison between measured and calculated excess pore pressure. Claye-Souilly/stage $310 / 4$.

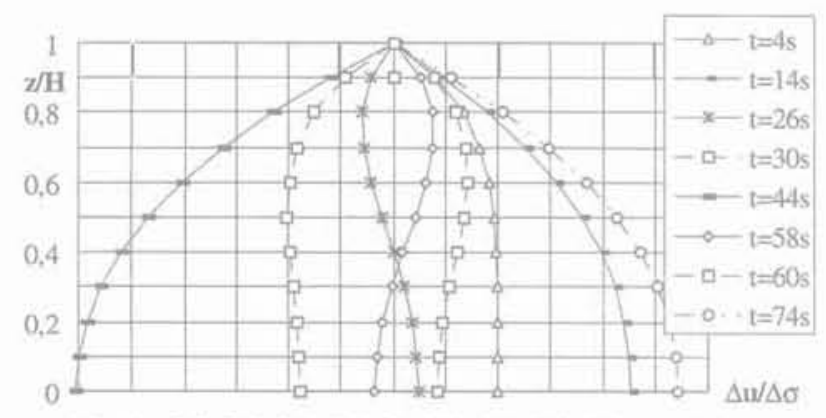

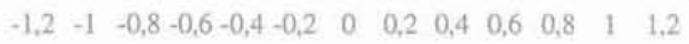

HG, 10 Évolution des isochrones de la surpression interstitielle.

Evolution of excess pore pressure isochrones.

La figure 10 représente les isochrones de la surpression interstitielle. Pendant le premier quart de période $(\mathrm{t}<15 \mathrm{~s})$ l'accroissement de $\mathrm{u}_{\mathrm{b}}(\mathrm{z}=0)$ est diminué par le drainage, d'où un rapport de pression $\Delta u / \Delta \sigma$ inférieur à 1 pour t égal à $14 \mathrm{~s}$. Dans le deuxième quart, le drainage accélère la décroissance de $\mathrm{u}_{\mathrm{p}}$ la pression est inférieure à zéro pour t égal à $30 \mathrm{~s}\left(\Delta \mathrm{u}_{\mathrm{h}}=-0,35 \Delta \sigma\right)$. Puis sa variation est ralentie dans le troisième quart tout en atteignant une valeur inférieure à $-\Delta \sigma\left(\Delta u_{\mathrm{b}}\right.$ $=-1,2 \Delta \sigma)$. Dans le quatrième quart, le drainage déve- 
loppe la croissance de $\mathrm{u}_{\mathrm{b}}$, la pression devient supérieure à zéro pour $\mathrm{t}$ égal à̀ $60 \mathrm{~s}\left(\Delta \mathrm{u}_{\mathrm{b}}=0,2 \Delta \sigma\right)$, puis il l'a ralentie dans le premier quart du cycle suivant, mais la pression atteint tout de même une valeur supérieure à $\Delta \sigma(\Delta u b=1,1 \Delta \sigma)$.

Dans la phase chargement, le point le plus instable se situe à $\mathrm{z} / \mathrm{H}=0$ sauf pour $\mathrm{t}=58 \mathrm{~s}$ et 60 s où il est à $\mathrm{z} / \mathrm{H}$ $=0,7$ et 0,6 respectivement.

\section{2}

\section{Étude du coefficient $n_{\mathrm{cf}}$}

En considérant le fluide incompressible (hypothèse de Terzaghi), c'est-à-dire avec un coefficient $n_{\text {f }}$ nul, il est possible de déterminer le coefficient de consolidation $\mathrm{c}_{\mathrm{y}}$ à partir de l'atténuation (cf. Fig. 2). Les résultats de ce calcul, représentés sur la figure 11, montrent des valeurs plus fortes que celles présentées dans le tableau III, mais qui se rapprochent de celles déterminées dans le cas des chargements "CRL » où $n_{\text {f }}$ est pris égal à zéro. Ceci montre que l'échantillon se comporte de la mème manière sous l'effet des deux types de chargement.

Or les valeurs de $n_{\text {, }}$ comme le montre le tableau III, sont bien différentes de zéro et croissantes en fonction de la période de chargement. Pour expliquer ceci nous utilisons les mesures de tassement car le dépouillement du chargement rend possible la détermination et de la compressibilité du squelette $m$ et de celle du fluide $\beta$. La figure 12 montre que le coefficient $m$ est constant en fonction de la période alors que $\beta$ croit considérablement. Cette augmentation de $\beta$ est d'autant plus surprenante que l'échantillon est soumis à plus de $300 \mathrm{kPa}$ de contre-pression.

\section{7}

\section{Conclusion}

La construction des ouvrages de génie civil implique des durées de chantier non négligeables. Le chargement du sol doit alors être représenté par un chargement fonction du temps. Il s'ensuit des évolutions de pression interstitielle qui ne peuvent pas être calculées en considérant l'ouvrage terminé comme situation de calcul. Cet article propose une solution de l'équation de la consolidation pour deux types de chargements continus: l'un appelé "CRL » à vitesse de chargement constante et l'autre cyclique sinusoïdal. Une étude expérimentale a été réalisée à l'œdomètre sur un limon en provenance du site de Claye-Souilly.

L'étude du premier type de chargement conduit aux mêmes conclusions que celles faites par Peignaud (1975), en particulier I'excès de pression interstitielle atteint un maximum qui dépend de la cadence de chargement. De plus, nous avons vérifié que la non-linéarité du comportement n'influe pas sur l'évolution de la pression interstitielle.

Le deuxième type de chargement fait apparaître au sein de l'échantillon des accroissements de pression interstitielle qui peuvent atteindre des niveaux supérieurs au chargement appliqué. Il est possible d'établir une solution analytique de l'équation de la consolidation proposée plus haut dans le cas de chargements sinusoïdaux. La solution montre explicitement les conditions pour lesquelles la surpression interstitielle

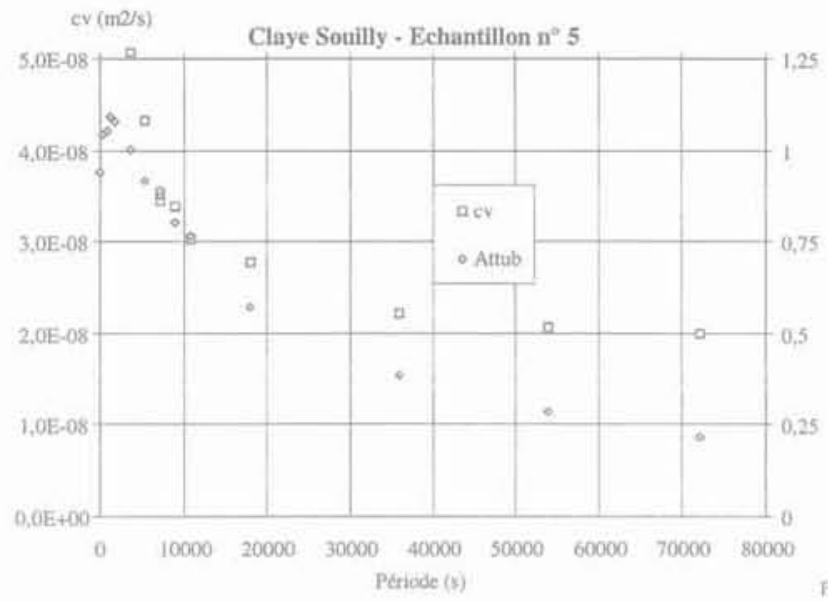

FG:11 Évolution de $c_{\mathrm{y}}$ et de l'atténuation en fonction de la période.

Echantillon de Claye-Souilly 5.

Evolution of $\mathrm{c}$, and the attenuation with respect to the period. Claye-Souilly 5.

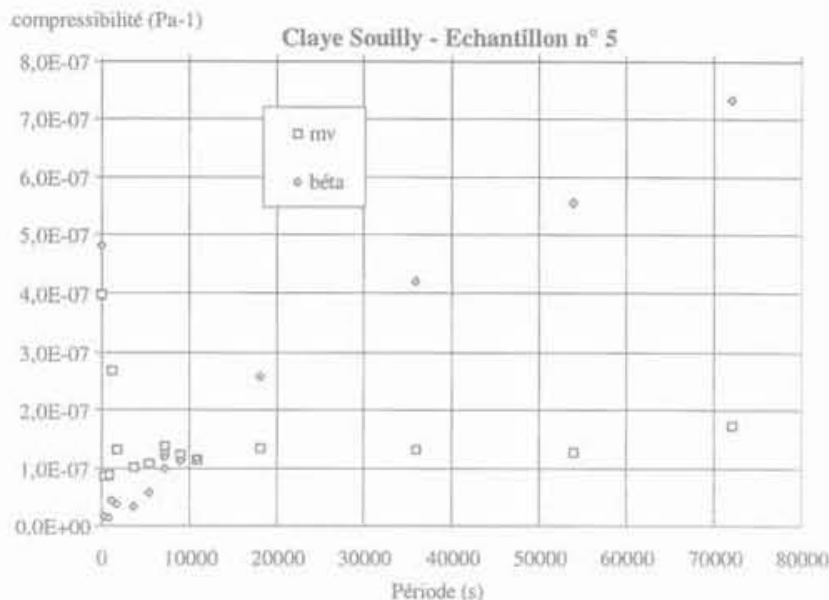

F1G. 12 Evolution de $m_{v}$ et de $\beta$ en fonction de la période.

Échantillon de Claye-Souilly 5 .

Evolution of $m_{y}$ and $\beta$ with respect to the period. Claye-Souilly 5

devient supérieure au chargement appliqué. Le sol doit être parfaitement saturé par un fluide peu compressible par rapport au squelette. La période de chargement doit être choisie en fonction de la hauteur de la couche de sol et du coefficient de consolidation c du matériau. Ces accroissements de pression doivent être pris en compte lors des calculs de stabilité d'ouvrages tels que silos ou réservoirs aux cycles de chargement très lents pouvant atteindre une période annuelle (Rahal et Vuez, 1997).

Le premier type de chargement ne traduit pas l'effet de la compressibilité du fluide interstitiel. Par contre le deuxième type permet de mesurer la compressibilité du squelette du sol et celle du fluide interstitiel. 
Les essais sur l'échantillon de Claye-Souilly ont été réalisés dans le cadre d'une étude sur les mesures de perméabilité dans les sols fins commanditée par l'ADEME.

\section{Bibliographie}

Aboshi H., Yoshikumi H., Murayamas S. a Constant loading rate consolidation test $x$. Solls and Fondation, vol. 10, n ${ }^{\circ} 1$, p. $43-56,1970$

Berry P.L., Poskitt T.J. - $*$ The consolidation of peat n. Geotechnique, vol. 22. p. $27-52,1972$

Ozisik N. - Heat conduction, John Wiley, 1980
Rahal A. - uttude de la consolidation unidimensionnelle d'un kaolin soumis a des chargements par paliers et sinusoídaux ». Thèse INSA de Rennes, 1993

Rahal A., Vuez A. - a Analysis of settlement and pore pressure induced by cyclic loading of a silo w. Journal of Geotechnic Engineering ASCE (accepté pour publication).
Vaid Y.P. - "Constant rate of loading nonlinear consolidation $v$. Soils and Foundations, vol. 25, n² 1, p. 105-108.

Vuez A., Rahal A. - « Cyclic loading for the measuring of soil consolidation parameters is. Proceedings of Settlement 94. Pub. N 40 ASCE, vol. 1, p. 760-774, 1994. 